\title{
A Study of ECG and Echocardiographic Findings in Chronic Obstructive Pulmonary Disease Patients in a Tertiary Care Hospital
}

\author{
Manasa Reddy Muskuํㅜ, Abdul Rafi Shaik², Roopa Reddy Mulamalla ${ }^{3}$ \\ ${ }^{1}$ Department of Pulmonary Medicine, Osmania Government General and Chest Hospital, Telangana, India. \\ ${ }^{2}$ Department of Pulmonary Medicine, Shadan Institute of Medical Sciences \& Research Centre, Telangana, India. \\ ${ }^{3}$ Department of Pulmonary Medicine and Critical Care, Vasavi Hospital \& Research Centre, Telangana, India.
}

\section{ABSTRACT}

\section{BACKGROUND}

COPD has considerable effects on cardiac functions. Most of the increased mortality and morbidity associated with COPD is due to cardiac involvement. We wanted to assess the electrocardiographic and echocardiographic findings in COPD, correlate these findings in assessing the severity and duration of the disease, analyse these findings in assessing right ventricular dysfunction and compare the results of clinical, electrocardiographic and echocardiographic findings in detecting right ventricular dysfunction in COPD.

\section{METHODS}

50 COPD patients fulfilling the inclusion criteria were recruited. They were staged by PFT and evaluated by ECG and echocardiography. Statistical analysis of correlation was done, and statistical significance was taken as $\mathrm{p}<0.05$.

\section{RESULTS}

Among the total of 50 cases selected for study $80 \%$ were males, $20 \%$ were females. On the basis of GOLD guidelines there were $8 \%, 44 \%, 36 \%, 12 \%$ mild, moderate, severe and very severe COPD cases respectively. Symptoms at presentation were cough with sputum (92\%), breathlessness (96\%), swelling of feet (24\%), fever (16 $\%)$, and decreased urine output (4\%). Physical signs at presentation were tachypnoea (68\%), loud P2 (24\%), parasternal heave $(20 \%)$, raised JVP (24\%), pedal oedema (16\%) and ascites (4\%). ECG findings analysis were P-pulmonale (46 $\%)$, RAD (38\%), RVH (34\%), low voltage complexes (30\%), poor progression of $\mathrm{r}$ wave (30\%) and incomplete RBBB (12\%). Correlation of all the above ECG findings showed statistical significance $(\mathrm{p}<0.05)$ with disease severity and disease duration $(\mathrm{p}<0.05)$. Echocardiographic findings were pulmonary hypertension (50\%), Cor pulmonale (44\%), RV dilatation (44\%), RA dilatation (32\%), RVH (24\%), interventricular septal motion abnormality (14\%), LVDD (16\%), LVSD (4\%) and RVSD (4 \%). All the above echocardiographic findings of RV dysfunction i.e. PAH, Cor pulmonale, RA dilatation, RVD, RVH and RVSD were statistically significant with disease severity and duration ( $\mathrm{p}<0.05$ ) along with LVDD, LVSD.

\section{CONCLUSIONS}

This study emphasises on early cardiac screening of all COPD patients which will be helpful in the assessment of the prognosis and will further assist in identifying the individuals likely to suffer increased morbidity and mortality.

\section{KEY WORDS}

Pulmonary Hypertension, Cor Pulmonale, RV Dysfunction, Echocardiography, ECG, Pulmonary Function Test

\author{
Corresponding Author: \\ Dr. Abdul Rafi Shaik, \\ 612 Mahara Block, \\ Garden Towers, Masab Tank, \\ Hyderabad-500028, \\ Telangana, India \\ E-mail:rafpulm@gmail.com
}

DOI: $10.14260 / j e m d s / 2020 / 612$

How to Cite This Article: Musku MR, Shaik AR, Mulamalla RR, et al. A study of ECG and echocardiographic findings in chronic obstructive pulmonary disease patients in a tertiary care hospital. J Evolution Med Dent Sci 2020;9(38):28102814, DOI: $10.14260 / \mathrm{jemds} / 2020 / 612$

Submission 13-06-2020,

Peer Review 14-08-2020,

Acceptance 21-08-2020,

Published 21-09-2020.

Copyright ( () 2020 Manasa Reddy Musku et al. This is an open access article distributed under Creative Commons Attribution License [Attribution 4.0 International (CC $B Y 4.0)]$ 


\section{BACKGROUND}

Chronic obstructive pulmonary disease is a common health problem seen world-wide. It carries significant morbidity and mortality. COPD has considerable effects on cardiac functions. Electrocardiography (ECG) and echocardiography can provide valuable information about cardiac disease and prognosis.

COPD is characterized by chronic airflow limitation and a range of pathological changes in the lungs. In addition COPD presents significant extrapulmonary effects and is associated with important comorbidities that may contribute to the disease severity. ${ }^{2}$ COPD is associated with significant extra pulmonary (systemic) effects among which cardiac manifestations are most common. Cardiovascular disease accounts for approximately $50 \%$ of all hospitalization and nearly one third of all deaths, if FEV1>50\% of predicted. In more advanced disease cardiovascular disease account for 20 $\%-25 \%$ of all deaths in COPD. ${ }^{3}$

COPD affects pulmonary blood vessels, right ventricle as well as left ventricle leading to the development of pulmonary hypertension (PH), Cor-pulmonale (COR-P), right ventricular dysfunction and left ventricular dysfunction. ${ }^{2}$ Different explanations have been suggested including systemic inflammation, vascular dysfunction and lung hyperinflation.

The level of Pulmonary Hypertension has a prognostic value in COPD patients demonstrated by several studies and a high degree of PH bears a poor prognosis and this has been observed in COPD patients receiving long-term oxygen therapy. Hypoxia is one of the major factor in bringing about ECG changes in COPD. Furthermore, pulmonary hypertension (PH) which is a frequent complication of COPD and the resulting right ventricular dysfunction are both predictive of survival in COPD.

Given the prognostic implications of cardiovascular disease in COPD its detection could serve as a guide to appropriate treatment and eventually improve survival. The ECG abnormalities are usually less pronounced in COPD than other forms of pulmonary hypertension because of the relatively modest degree of pulmonary hypertension and effects of hyperinflation. ${ }^{4}$ 2D echocardiography can be used to assess right ventricular dimensions and wall thickening and right ventricular volume overload in patients with COPD and also the presence of pulmonary artery hypertension. Many studies hypothesised that a high proportion of COPD patients might present clinically silent echocardiographic abnormalities and that these cardiac alterations could constitute a clinically relevant trait in COPD.

In this study, we made an attempt to see the importance of ECG and echocardiography in the evaluation of COPD patients and early recognition of complications.

\section{Objectives}

1. To assess the electrocardiographic and echocardiographic findings in COPD.

2. To correlate these findings in assessing the severity and duration of the disease.

3. To analyse these findings in assessing right ventricular dysfunction.

4. To compare the results of clinical, electrocardiographic and echocardiographic findings in detecting right ventricular dysfunction in COPD.

\section{METHODS}

The present study proposal was approved by the institutional ethical committee of MNR Hospital, Sangareddy. 50 patients who fulfilled the inclusion \& exclusion criteria were selected. Data has been collected over a period of 2 years. It is a crosssectional study.

\section{Inclusion Criteria}

Adult male and female COPD patients aged more than 40 years with informed and written consent.

\section{Exclusion Criteria}

- Patients with known respiratory problems other than COPD

- $\quad$ The patients with history of cardiac diseases like ischemic heart disease, rheumatic heart disease, valvular heart diseases, congenital heart disease, and others. Patients who were immune compromised.

- Very poor echogenic subjects in whom meaningful echocardiographic examination could not be performed were also excluded.

A detailed clinical history was taken, and examination was done in all selected patients. They were subjected to routine investigations including complete blood count, lipid profile, blood sugar, blood urea, serum creatinine and so on as needed. Pulmonary function tests were done in all patients and patients were graded according to the severity of COPD with guidelines given by GOLD. ${ }^{1}$ The patients were subjected to radiological examination, 12 lead ECG and 2D echo were done to assess the severity of right ventricular dysfunction. Various observations in the study were analysed and correlated.

\section{Statistical Analysis}

Data was tabulated, frequency and percentage of cases was analyzed. p values were calculated using Fischer's exact probability test wherever necessary and SPSS 25 software was used to analyze. $\mathrm{p}<0.05$ was considered to be statistically significant.

\section{RESULTS}

50 patients were included in the study. Mean age SD was 61.94 \pm 7.39 years. In the study, majority of male patients (72\%) were smokers. All females (20\%) were exposed to biomass fuel. The mean duration of smoking observed was $27.2+5.21$ years. Majority of smokers (54\%) had history of smoking more than 20 pack years. The mean duration of disease was $5.07+3.72$ years, range $1-16$ years. Maximum number of patients (56\%) had symptoms of 1-5 years of duration and patients in more than 10 years of disease were only $12 \%$. Most of the patients had cough with sputum (92 \%) and breathlessness (96\%), swelling of feet (24\%), fever (16\%), decreased urine output ( $4 \%$ ). The most common sign at presentation is tachypnoea in $68 \%$ followed by $24 \%$ had loud 
P2 suggestive of $\mathrm{PH}, 20 \%$ of the patients had parasternal heave, the clinical evidence of right ventricular hypertrophy. Evidence of congestive cardiac failure like raised JVP in $24 \%$, pedal oedema in $16 \%$ and ascites in $4 \%$ cases. $20 \%$ of the patients had cyanosis which is evidence of a hypoxic state.

A wide range of COPD severity was found, although the majority of patients had moderate to severe disease with a mean post-bronchodilator forced expiratory volume in $1 \mathrm{~s}$ (FEV1) of $53.11 \pm 15.29 \%$ of predicted. In chest X - ray, $76 \%$ had emphysema, $64 \%$ had increased bronchovascular marking suggestive of chronic bronchitis. X - ray evidence of pulmonary hypertension i.e. prominent right descending pulmonary artery in $24 \%$ and cardiomegaly in $16 \%$ of patients were observed.

\begin{tabular}{|ccc|}
\hline Disease Severity (FEV1 of \% Predicted) & No. of Patients & Percentage \\
$>80$ (Mild ) & 4 & $8 \%$ \\
$50-80$ (Moderate) & 22 & $44 \%$ \\
$30-50$ (Severe) & 18 & $36 \%$ \\
$<30$ (Very Severe) & 6 & $12 \%$ \\
\hline Table 1. Severity of the Disease among COPD Patients \\
\hline
\end{tabular}

\begin{tabular}{|ccc|}
\hline ECG Changes & No. of Patients & Percentage \\
p-Pulmonale & 23 & $46 \%$ \\
Low Voltage Complexes & 15 & $30 \%$ \\
Right Axis Deviation & 19 & $38 \%$ \\
Poor Progression of r Wave & 15 & $30 \%$ \\
Incomplete RBBB & 6 & $12 \%$ \\
Right Ventricular Hypertrophy & 17 & $34 \%$ \\
\hline Table 2. Analysis of ECG Changes among COPD Patients \\
\hline
\end{tabular}

\begin{tabular}{|ccc|}
\hline Echocardiographic Changes & No. of Patients & Percentage \\
\hline Right Atrial Dilatation & 16 & $32 \%$ \\
Right Ventricular Dilatation & 22 & $44 \%$ \\
Right Ventricular Hypertrophy & 12 & $24 \%$ \\
Interventricular Septum Motion Abnormality & 7 & $14 \%$ \\
\hline Pulmonary Hypertension & 25 & $50 \%$ \\
Cor Pulmonale & 22 & $44 \%$ \\
LVDD & 8 & $16 \%$ \\
LVSD & 2 & $4 \%$ \\
RVSD & 2 & $4 \%$ \\
\hline Table 3. Analysis of Echocardiographic Changes among COPD Patients \\
\hline
\end{tabular}

In this study the correlation of ECG findings with severity of the disease showed that the findings of ' $p$ ' pulmonale, RAD (right axis deviation), poor r-wave progression, incomplete RBBB and RVH correlate significantly with severity of the disease. $(p<0.05)$. Low voltage complexes are not correlated significantly with severity of the disease. All the above ECG findings except low voltage complexes and poor progression of $r$ - wave had significant correlation with duration of the disease $(\mathrm{p}<0.05)$. All the above echocardiographic findings of RV dysfunction i.e. PAH, Cor pulmonale, RA dilatation, RVD, RVH and RVSD linear correlated significantly with disease severity $(p<0.05)$ and LVDD, LVSD were also correlated significantly with disease severity. All the above echocardiographic findings were also correlated significantly with the duration of disease $(\mathrm{p}<0.05)$.

\section{DISCUSSION}

COPD is a male dominant disease, the high prevalence in males is due to higher prevalence of smoking in this gender and also males are more susceptible to smoking than females. In present study $80 \%$ of the population were males, $20 \%$ were females. Male to female ratio is 4:1. Use of biomass and coal as their main source of energy for cooking, heating and other household needs of possibly a strong risk factor for development of COPD among female patient. This study is similar to other studies done by Suma et al, ${ }^{5}$ Swathi talari et al, 6 dhadke et al, 7 Jatav VS et al. ${ }^{8}$

COPD is a disease of late adulthood. As the age advances the lung function (FEV1) declines and other risk factors add to the disease process. Patients between 50 - 70 years form the maximum number of patients admitted mainly because of the longer duration of tobacco exposure and repeated respiratory tract infections, which would have compromised their quality of life. This study age distribution is similar to studies conducted by Dhadke et al (38 \%), ${ }^{7}$ Jatav VS et al (70 \%), ${ }^{8}$ Dave L et al, ${ }^{9}$ Lokesh et al (40\%), ${ }^{10}$ Rajan Chaudhari et al (70\%), ${ }^{11}$ where maximum number of patients were present in the 6 th and 7 th decade.

In the remaining male patients ( $8 \%$ ) who did not smoke, no definite cause can be established hence passive smoking and environmental factors like outdoor pollution was considered to be the possible aetiology for COPD in them. In the studies by Suma et $\mathrm{al}^{5}$ and Jatav VS et al ${ }^{8}$ mean duration of smoking was 23.2 years and 25.06 pack years respectively which is similar to the present study (27.52 pack years).

The mean duration of symptoms was $5.07+3.72$ years. This is similar to the studies conducted by Suma et al, 5 Jatav VS et $\mathrm{al}^{8}{ }^{8}$ Dave $\mathrm{L}$ et $\mathrm{al}^{9}$, Lokesh et $\mathrm{al}^{10}$ Rajan chaudhari et al ${ }^{11}$ in which the mean duration of symptoms were $5.71+4.98$ years, 7.58 years, $6.12+4.5$ years, 5.78 years, 7.58 years respectively.

The exact threshold for the duration/ intensity of cigarette smoking that will results in COPD usually varies from one individual to another.

Breathlessness is the symptom that commonly causes the patient to seek medical attention and is usually the most disabling of these symptoms. Close questioning usually reveals the presence of a "smokers cough" with scanty mucoid sputum, mainly in the morning for many years. This study correlates with Suma et al, ${ }^{5}$ Jatav et al, ${ }^{8}$ Lokesh et al ${ }^{10}$ studies.

Physical signs and chest $\mathrm{x}$-ray features of this study correlates with Suma et al, ${ }^{5}$ Dhadke et al, ${ }^{7}$ Jatav et al ${ }^{8}$ studies.

The mean FEV1 was $53.11+15.29 \%$ of predicted range. In this study most of the population were moderate and severe COPD (80 \%) this study findings correlates with Suma et al ${ }^{5}$, Jatav et $\mathrm{al}^{8}$ studies who also used GOLD guidelines for staging of severity of COPD.

ECG changes observed in this study correlates with Suma et $\mathrm{al},{ }^{5}$ dhadke et al, ${ }^{7}$ Lokesh $\mathrm{S}$ et al. ${ }^{10}$ Ghosh et al,12 V.V. Rao et al, ${ }^{13}$ Rachaiah et al ${ }^{14}$ studies.

ECG changes are mainly due to change in haemodynamics of pulmonary vasculature secondary to hypoxia. The presence of increased air (hyperinflation of lungs) between the heart and recording electrodes has a dampening effect, leading to reduced amplitude of the QRS complexes. Thus we can say that development of ECG findings demonstrate that the COPD is progressive and is of higher grade.

In this study $4 \%$ of cases had cardiac arrythmias (atrial fibrillations and ectopics) COPD predisposes patients to cardiac arrhythmias and pulmonary hypertension. PH causes right ventricular strain and stretching of the right atrium which predisposes patients to arrhythmias. Arrythmias are 
sometimes the result of the underlying disease process but may also occur as side effects of the drugs used to treat the disease. Arrythmias are mostly supraventricular in origin. Ventricular extra systoles and ventricular tachycardia may also occur in COPD. 15

In this study the correlation of ECG findings with severity of the disease was studied as the hypoxia in COPD is directly proportional to severity of airway obstruction. This study correlates with Suma et al, ${ }^{5}$ Jatav VS et al, ${ }^{8}$ Dave L et al, ${ }^{9}$ Lokesh $\mathrm{S}$ et al, ${ }^{10} \mathrm{Ghosh}$ et al ${ }^{12}$ Rachakonda et al, ${ }^{16}$ Janak chokshi et al ${ }^{17}$ studies.

In this study, P-pulmonale, incomplete RBBB, RVH, RAD had significant correlation with duration of symptoms $(\mathrm{p}<$ 0.05). The other findings low voltage complexes and poor progression of $r$ wave did not show any correlation with the duration of symptoms. This study correlates with Suma et al, 5 Lokesh et al,10 Rajan Chaudhari et al, ${ }^{11}$ Sachadeva et al ${ }^{18}$ studies.

Most common echocardiographic finding was pulmonary hypertension. Pulmonary arterial hypertension (PAH) which is defined as pulmonary arterial systolic pressure (PASP) $>30$ mmHg was observed in $50 \%$ cases. In the pulmonary circulation in patients with COPD significant structural changes occur. Hypoxemia and chronic ventilator insufficiency is associated with early evidence of medial hypertrophy and intimal thickening in the smaller branches of the pulmonary arteries. Along with these pathological changes, pulmonary vasoconstriction arising from the presence of alveolar hypoxemia, pulmonary vascular bed destruction, changes in intrinsic pulmonary vasodilator substances (decrease in prostacyclin synthase), decrease in eNOS (endothelial nitric oxide synthase) and increase in ET1 (endothelin 1) lead to remodeling, increase in blood viscosity and alteration in respiratory mechanics. All the above-mentioned changes lead to a significant increase in pulmonary vascular resistance because of which $\mathrm{PH}$ arises. ${ }^{19}$ Other echocardiographic findings noted in our study were cor pulmonale in $44 \%$ cases, $\mathrm{RV}$ dilatation in $44 \%$, RA dilatation in $32 \%$, RVH in $24 \%$, LVDD in $16 \%$, Interventricular septal motion abnormality in $14 \%$, RVSD in $4 \%$ and LVSD in $4 \%$ of cases. This study correlates with Suma et al, ${ }^{5}$ Dhadke et al, ${ }^{7}$ Dave L et al, ${ }^{9}$ V.V. Rao et al, ${ }^{13}$ Rachakonda et al, ${ }^{16}$ Jatav VS et al ${ }^{20}$ studies.

In patients with COPD hypoxic vasoconstriction leads to right ventricular hypertrophy and dilation which leads to development of right heart failure with systemic congestion and inability to adapt to the peripheral demand on exercise ${ }^{19}$. Numerous studies have examined the correlation between right ventricular systolic pressure (RVSP) as estimated by Doppler echocardiography and RVSP as directly measured during right heart catheterization and most of the studies reported a relatively tight correlation (the $r$ value ranged from $0.57-0.95$ ).

In the present study, the incidence of all the ECHO findings increased as the severity of the disease increased. Other studies (Suma et $\mathrm{al}^{5}$ Jatav et $\mathrm{al}^{20}$ ) correlating the ECHO findings with severity of the disease have also made similar observations and also have given different explanations for their observation. Some studies indicate that LV function remains normal in persons with COPD where as others suggest that LV dysfunction may be present. Abnormal LV function in patients with COPD may be due to factors such as hypoxemia and acidosis, coronary artery disease, ventricular interdependence RV and LV share a common septum, hence $\mathrm{RV}$ dilatation may lead to bulging of the septum into the $\mathrm{LV}$ which would in turn increase LV end-diastolic pressure, decrease venous return, and diminish LV stroke volume and cardiac output and large swings in intrathoracic pressure (negative pleural pressure) would increase Ppa and diminish LV stroke volume due to ventricular interdependence, negative pleural pressures may also increase LV after load.21 LVDD was seen in COPD patients with normal pulmonary arterial pressure and its incidence increased with increase in right ventricular after load. Left diastolic dysfunction in COPD patients may be due to chronic hypoxemia leading to myocardial relaxation abnormalities, lung hyperinflation and distension thereby leading to increased stiffness of the parietal pleura and hence the wall of cardiac fossa leading to an extra load on the ventricle and also due to ventricular interdependence. ${ }^{21}$

The echocardiographic findings of $\mathrm{PH}$, RA dilatation, RV dilatation, LVDD, LVSD, RVSD, Interventricular septal motion abnormality, RVH and cor pulmonale correlated significantly with the duration of disease $(p<0.05)$. This can be explained by the fact that longer the duration of the disease higher is the chances that the patient has developed pulmonary hypertension and cor pulmonale. This study correlates with the study conducted by Suma et al. ${ }^{5}$

In this study, a diagnosis of cor pulmonale could be made in $24 \%$ of patients by clinical, $34 \%$ by electrocardiographic and $44 \%$ by echocardiographic methods. This shows that echocardiography can detect more number of patients with cor - pulmonale in COPD and is similar to previous studies. This is because clinical signs of RV dysfunction are difficult to detect in COPD due to lung hyperinflation and posterior rotation of heart. ECG criteria for detecting RVH have a reasonably high specificity but relatively low sensitivity. Most studies report that adequate examination can be obtained in more than $70 \%$ of the patients. Many studies (Suma et al, 5 Rachakonda et al,16 Gupta NK et al,21 Satish Kinagi et al,22 Vikram B Vikhe et $\mathrm{al}^{23}$ ) have proved that echocardiography is more sensitive than electrocardiography in detecting RV dysfunction in COPD.

\section{CONCLUSIONS}

The study shows high prevalence of cardiac comorbidities such as $\mathrm{PAH}$, cor pulmonale, RV dysfunction and LV dysfunction in COPD patients. The severity of complications increases with severity of COPD and makes a linear relation. ECG can be used for screening of COPD patients with the most parameters observed. But ECHO may be the better modality for detection of cardiac dysfunction as compared to ECG. Although ECG and ECHO are less informative as compared to pulmonary artery catheterisation, ECHO being non-invasive and easily available, can be routinely recommended among COPD patients. Echocardiographic examination is reliable in COPD patients with PAH instead of repeated cardiac catheterization. This study puts emphasis on early cardiac screening of all COPD patients which will be helpful in the 
assessment of the prognosis and will further assist in identifying the individuals likely to suffer increased morbidity and mortality.

Financial or Other Competing Interests: None.

\section{REFERENCES}

[1] Global initiative for chronic obstructive pulmonary disease-global strategy for diagnosis, management, and prevention of chronic obstructive pulmonary disease. 2019. http.//www.goldcopd.com

[2] Kaushal M, Shah PS, Shah AD, et al. Chronic obstructive pulmonary disease and cardiac comorbidities. A cross sectional study. Lung India 2016;33(4):404-9.

[3] Thomason MJ, Strachan DP. Which spirometric indices best predict subsequent death from chronic obstructive pulmonary disease? Thorax 2000;55(9):785-8.

[4] McLaughlin VV, Rich S. Cor-pulmonale. Chap- 54. In: Braunwald E, Zipes DP, Libby P, eds. Heart disease: a text book of cardiovascular medicine. $6^{\text {th }}$ edn. Philadelphia: W.B. Saunders Company 2001:1936-54.

[5] Suma KR, Srinath PS. Electrocardiographic and echocardiographic changes in chronic obstructive pulmonary disease (COPD) of different grades of severity. J Evolution Med Dent Sci 2015;4(30):5093-101.

[6] Swathi T, Vundela DN, Kucherlapati D, et al. The study of electrocardiographic and echocardiographic changes in COPD. J Evolution Med Dent Sci 2018;7(02):135-41.

[7] Dhadke VN, Dhadke SV, Raut N. Clinical profile in chronic obstructive pulmonary disease patients and their evaluation with spirometry and 2D echo. Inter J Curr Res 2015;7(2):12480-8.

[8] Jatav VS, Meena SR, Jelia S, et al. Electrocardiographic characteristics of patients with chronic obstructive pulmonary disease and its correlation with disease severity. Int J Adv Med 2017;4(2):514-8.

[9] Dave L, Rajoriya V, Dubey TN, et al. Evaluation of BODE index as a predictor of pulmonary hypertension in COPD patients. Ann Int Med Den Res 2015;1(3):213-17.

[10] Lokesh S, Naik L. Cardiac evaluation of patients with chronic obstructive pulmonary disease using electrocardiogram with special reference to severity and duration in a tertiary care centre. Inter J Contem Med Surg Radiol 2018;3(3):C101-4.

[11] Chaudhari R, Shrimali L. Study of clinical electrocardiographic and echocardiographic profile in patients with chronic obstructive pulmonary disease. Int J Res Med Sci 2018;6(5):1716-20.

[12] Ghosh SK, Sharma HS. A study on ECG changes in COPD patients. IJSR - Inter J Sci Res 2017;6(2):190-1.

[13] Rao VV, Eswaramma, Soujanya. Study of cardiovascular changes in COPD by ECG \& 2D echo and correlation with duration and severity of COPD. Sch J App Med Sci 2016;4(12D):4430-8.

[14] Rachaiah NM, Rachaiah JM, Krishnaswamy RB. A correlative study of spirometric parameters and ECG changes in patients with chronic obstructive pulmonary disease. Int J Biol Med Res 2012;3(1):1322-6.

[15] Reddy BSK, Lokendranath G, Rao RP. Electrocardiographi changes in chronic obstructive pulmonary disease. J of Evidence Based Med \& Hlthcare 2014;1(3):111-7.

[16] Rachakonda R, Beri S, Kalyankumar PV. Study of ECG and echocardiographic findings in COPD patients in a tertiary care centre. J Evolution Med Dent Sci 2016;5(24):127680.

[17] Chokshi J, Patil A, Purohit N, et al. Study of electrocardiographic and echo cardiographic profile of chronic obstructive pulmonary disease patients. Inter J Sci Res 2014;3(10):385-6.

[18] Sachdeva GS, Bhagat A, Saini V, et al. Electrocardiographic changes in patients of COPD and their clinical correlation with disease severity. Int J Curr Res Biol Med 2018;3(2):40-7.

[19] Gupta NK, Agrawal RK, Srivastav AB, et al. Echocardiographic evaluation of heart in chronic obstructive pulmonary disease patients and its corelation with severity of disease. Lung India 2011;28(2):105-9.

[20] Jatav VS, Meena SR, Jelia S, et al. Echocardiographic findings in chronic obstructive pulmonary disease and correlation of right ventricular dysfunction with disease severity. Int J Adv Med 2017;4(2):476-80.

[21] Murugan AS, Aravind C, Muruga BB, et al. Echocardiographic features in COPD patients. Int J Pharm Biol Sci 2016;6(4):9-12.

[22] Kinagi S, Patil S, Afiya S, et al. Analysis of chronic obstructive pulmonary disease with clinical parameters, ECG, and echo. J Evolution Med Dent Sci 2014;3(57):12864-80.

[23] Vikhe VB, Shende PS, Patil RS, et al. Cardiovascular complications in chronic obstructive pulmonary disease with reference to $2 \mathrm{~d}$ echocardiography findings. National J Med Res 2013;3(4):385-8. 